\title{
Evaluation of Concentrated Oblique Load at the Apex of a Wedge by the Method of Caustics
}

\author{
G.A. Papadopoulos* \\ National Technical University of Athens, Department of Mechanics,5, Heroes of Polytechnion Av., GR-157 73, \\ Zografou, Athens, Greece
}

\begin{abstract}
The experimental method of caustics was used for the evaluation of the stress-singularities created by concentrated oblique load applied at the apex of a wedge or on the horizontal straight boundary of a half-plane. The wedge was considered to be elastic, isotropic and under generalized plane stress conditions. According to the method of caustics, the light rays impinging normally at the thin plate are partly reflected from either the front or the rear faces of the plate. The reflected rays are deviated because of the important constraint of the plate at the vicinity of the applied concentrated load and the significant variation of the refractive index there. The deviated light rays, when projected on a reference screen, are concentrated along a singular curve which is strongly illuminated and forms a caustic. It was proved that the shape and size of the caustic depend on the stress singularity at the point of application of the load. The parametric equations of caustics created by such a singularity were studied in relation with the loading mode of the wedge. Thus, by measuring the dimensions of the caustic, one can evaluate the state of stress at the singularity. The experimental evidence, based on the above method on Plexiglas (PMMA) wedges, corroborated existing elastic theories concerning the order of singularities for concentrated loads. The relations for the calculation of the load, the components of stress and the concentration factor based on the geometrical characteristic of caustics, which were reflected from the front face of the wedge, were given.
\end{abstract}

Keywords: Caustics, wedges, concentrated load, concentration factors, contact problem.

\section{INTRODUCTION}

The optical method of caustics is suitable for the experimental study of singularities in stress fields created either by geometric discontinuities or by loading. For many crack problems, the stress intensity factors at the crack-tip were calculated by caustics [1-3]. Contact and wedge problems have many practical and important applications [4]. The stress distribution problem in a semi infinite plate under generalized plane stress conditions, which is loaded by a concentrated load applying at a point of the straight boundary was solved by Flamant [5], while Boussinesq [6] solved the same problem for the case of any inclined load.

For the solution of such problems, besides mechanical analysis, the experimental method of caustics can also be applied. Caustics has been applied to the study of the singular stress fields developed near concentrated or uniformly distributing loads which are applied along straight boundaries $[7,8]$, the load sharing in roller-bearings $[9,10]$, in multiple gear tooth contact $[11,12]$ and in study of vertical concentrated load in wedges [13]. Also, three-dimensional wedge problems, bimaterial wedges in antiplane shear deformation and rubber wedges in large strain analysis were studied [14-16]. The optical method of caustics has been applied to study of the singular stress fields near holes $[17,18]$ and in the contact problems of anisotropic materialas [19].

*Address correspondence to these authors at the National Technical University of Athens, Department of Mechanics,5, Heroes of Polytechnion Av., GR-157 73, Zografou, Athens, Greece; Tel: +30 2107721228 ;

Fax: +30 210 7721302; E-mail: gpad@central.ntua.gr
The aim of the present work is to give the parametric equations of the caustics in wedges made of isotropic materials for the case of a concentrated oblique load at the apex of the wedge. Also, the relations of calculation of the concentrated oblique load at the apex of wedge, the components of stress at any point and the concentration factors of the stresses based on the diameter and the opening displacement of the caustic were given.

\section{CONCENTRATED OBLIQUE LOAD AT THE APEX OF THE WEDGE}

Concentrated oblique load $P$ per unit thickness of the wedge (compressive load) was applied on the direction which is limited by the angle $\omega$ with the axis of the wedge (Fig. 1). The thickness of the wedge was taken as unity. Resolving the oblique force $\mathrm{P}$ into two components, $P \cos \omega$ vertically and $P \sin \omega$ horizontally (Fig. 1), the conditions along the faces, $\theta= \pm \phi$, of the wedge are satisfied by the stress components which are derived from the stress function [4]:

$\Phi=-\frac{P}{2} A r \theta \sin \theta-\frac{P}{2} B r\left(\frac{\pi}{2}+\theta\right) \sin \left(\frac{\pi}{2}+\theta\right)$

with:

$$
A=\frac{\cos \omega}{\phi+\frac{1}{2} \sin 2 \phi}, \quad B=\frac{\sin \omega}{\phi-\frac{1}{2} \sin 2 \phi}
$$

where $\phi$ is the angle of wedge and $r, \theta$ are the polar coordinates. 


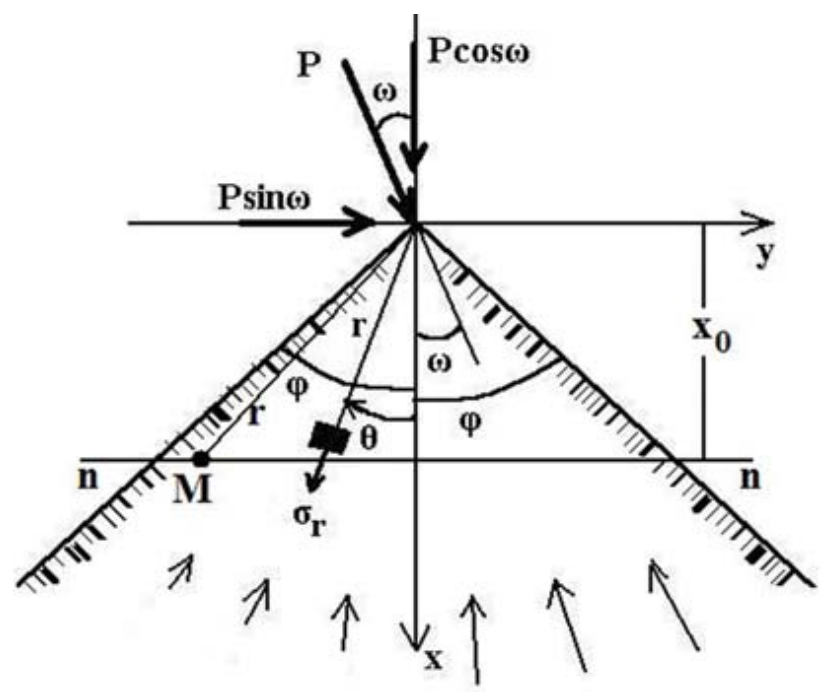

Fig. (1). Geometry of a symmetrical wedge of angle $2 \varphi$.

The stress components are derived from equation (1) as:

$$
\begin{aligned}
\sigma_{r r} & =\frac{1}{r} \frac{\partial \Phi}{\partial r}+\frac{1}{r^{2}} \frac{\partial^{2} \Phi}{\partial \theta^{2}}=-P A \frac{\cos \theta}{r}+P B \frac{\sin \theta}{r} \\
& =-k_{1} \frac{\cos \theta}{r}+k_{2} \frac{\sin \theta}{r} \\
\sigma_{\theta \theta} & =\frac{\partial^{2} \Phi}{\partial r^{2}}=0, \tau_{r \theta}=-\frac{\partial}{\partial r}\left(\frac{1}{r} \frac{\partial \Phi}{\partial \theta}\right)=0
\end{aligned}
$$

The constants:

$$
\begin{aligned}
& k_{1}=P A=\frac{P \cos \omega}{\varphi+\frac{1}{2} \sin 2 \varphi} \\
& k_{2}=P B \frac{P \sin \omega}{\varphi-\frac{1}{2} \sin 2 \varphi}
\end{aligned}
$$

are the stress concentration factors.

By taking $\phi=\pi / 2$ and $\omega=0$ the solution for a semiinfinite plate was given which has been studied by the method of caustics and photoelasticity method $[9,10]$.

For $\omega=0$ (vertical force at $O$, Fig. 1) the stress function and the radial stress are [13]:

$$
\begin{aligned}
& \Phi=-\frac{P}{2} A r \theta \sin \theta \\
& \sigma_{r r}=-k_{1} \frac{\cos \theta}{r}
\end{aligned}
$$

with:

$$
A=\frac{1}{\phi+\frac{1}{2} \sin 2 \phi} \text { and } k_{1}=P A=\frac{P}{\varphi+\frac{1}{2} \sin 2 \varphi}
$$

and for $\omega=\pi / 2$ (horizontal force at O, Fig. 1) the stress function and the radial stress are:

$$
\begin{aligned}
& \Phi=-\frac{P}{2} B r\left(\frac{\pi}{2}+\theta\right) \sin \left(\frac{\pi}{2}+\theta\right) \\
& \sigma_{r r}=k_{2} \frac{\sin \theta}{r}
\end{aligned}
$$

with:

$$
B=\frac{1}{\phi-\frac{1}{2} \sin 2 \phi} \text { and } k_{2}=P B=\frac{P}{\varphi-\frac{1}{2} \sin 2 \varphi}
$$

\section{EXPERIMENTAL METHOD OF CAUSTICS}

The light rays reflected from the rear or front face of the specimen or transmitted through the specimen form wave fronts [1-3]:

$$
S(x, y, z)=\text { const } \text {. }
$$

If $s(x, y)$ expresses the optical path of the light ray between two planes parallel to the middle plane of the loaded plate and lying at the faces of the plate, then the relation:

$S(x, y, z)=z-s(x, y)=$ const .

is valid and:

$\operatorname{grad} S(x, y, z)=\mathbf{k}-\frac{\partial s}{\partial x} \mathbf{i}-\frac{\partial s}{\partial y} \mathbf{j}$

where $\mathbf{i}, \mathbf{j}, \mathbf{k}$ are the unit vectors of the $(O x, y, z)$ Cartesian coordinates system. For optically isotropic materials and for the first light ray reflected from the rear face (r) or from the front face (f) of the specimen the deviation of the light rays at a distance $\mathrm{z}_{0}$ from the middle plane of the specimen, is expressed by the vector $\boldsymbol{w}_{r, f}$ on the plane $z=z_{0}$ and according to the theory of Econal [20], is given as:

$$
\mathbf{w}_{r, f}=-\frac{\varepsilon z_{0} d c_{r, f}}{\lambda_{m}} \operatorname{grad}\left(\sigma_{1}+\sigma_{2}\right)
$$

where $d$ is the thickness of the specimen, $c_{r, f}$ are the stressoptical constants, $\varepsilon=1$ for reflected light rays from the front (f) face and $\varepsilon=2$ for reflected light rays from the rear (r) face of the specimen and $\lambda_{m}$. is the magnification ratio, which for divergent light beam is given by the relation:

$$
\lambda_{m}=\frac{z_{0}+z_{i}}{z_{i}}
$$

where $z_{0}$ is the distance between reference-plane and specimen and $z_{i}$ is the distance between specimen and the light beam focus. The stress-optical constant $c_{\mathrm{f}}$ is $c_{f}=v / E$, where $v$ is the Poisson's ratio and $E$ is the modulus of elasticity of the specimen material. The sum of the stresses $\sigma_{1}+\sigma_{2}$ is given by:

$\sigma_{1}+\sigma_{2}=\sigma_{r r}+\sigma_{\theta \theta}=-P A \frac{\cos \theta}{r}+P B \frac{\sin \theta}{r}=-k_{1} \frac{\cos \theta}{r}+k_{2} \frac{\sin \theta}{r}$ 
Then, the Rel.(13) becomes:

$$
\begin{aligned}
& \mathbf{w}_{r, f}=C_{r, f} \operatorname{grad}_{(x, y)}\left[A \frac{1}{r} \cos \theta+B \frac{1}{r} \cos \left(\frac{\pi}{2}+\theta\right)\right]= \\
& C_{r, f}\left\{-A \frac{1}{r^{2}}[\cos 2 \theta \mathbf{i}+\sin 2 \theta \mathbf{j}]+B \frac{1}{r^{2}}[\sin 2 \theta \mathbf{i}-\cos 2 \theta \mathbf{j}]\right\}
\end{aligned}
$$

where:

$$
C_{r, f}=-\frac{\varepsilon z_{0} d c_{r, f} P}{\lambda_{m}}, c_{f}=\frac{v}{E}
$$

The parametric equations of the caustic are:

$$
\mathbf{W}_{r, f}=\mathbf{r}+\mathbf{w}_{r, f}=r \cos \theta+i r \sin \theta+\mathbf{w}_{r, f}
$$

or:

$$
X_{r, f}=r \cos \theta \pm C_{r, f} r^{-2}[A \cos 2 \theta-B \sin 2 \theta]
$$

$Y_{r, f}=r \sin \theta \pm C_{r, f} r^{-2}[A \sin 2 \theta+B \cos 2 \theta]$

where $r$ is the radius of the initial curve of the caustics and $\theta$ takes values in the region $-\phi \leq \theta \leq+\phi$. For divergent light beam, the sign (-) corresponds to the caustics (r) (caustics which are formed by the reflected light rays from the rear face (r) of the specimen) and the sign (+) corresponds to the caustic (f) ( caustics which are formed by the reflected light rays from the front face (f) of the specimen).

The initial curve $r$ of the caustic is determined by setting the functional determinant Jacobian J equal to zero:

$J=\frac{\partial\left(X_{r, f} Y_{r, f}\right)}{\partial(r, \theta)}=0$

Then, the initial curve is:

$r_{0}=r=\left(2 C_{r, f} \sqrt{A^{2}+B^{2}}\right)^{1 / 3}$

By substituting equation (21) into equations (18), (19) the parametric equations of the caustics becomes:

a) caustics (f):

$X_{f}=\lambda_{m}\left(2 C_{f} \sqrt{A^{2}+B^{2}}\right)^{1 / 3}\left[\cos \theta+\frac{1}{2 \sqrt{A^{2}+B^{2}}}(A \cos 2 \theta-B \sin 2 \theta)\right]$
$Y_{f}=\lambda_{m}\left(2 C_{f} \sqrt{A^{2}+B^{2}}\right)^{1 / 3}\left[\sin \theta+\frac{1}{2 \sqrt{A^{2}+B^{2}}}(A \sin 2 \theta+B \cos 2 \theta)\right](22)$

b) caustics (r):

$X_{r}=\lambda_{m}\left(2 C_{r} \sqrt{A^{2}+B^{2}}\right)^{1 / 3}\left[\cos \theta-\frac{1}{2 \sqrt{A^{2}+B^{2}}}(A \cos 2 \theta-B \sin 2 \theta)\right]$

$Y_{r}=\lambda_{m}\left(2 C_{r} \sqrt{A^{2}+B^{2}}\right)^{1 / 3}\left[\sin \theta-\frac{1}{2 \sqrt{A^{2}+B^{2}}}(A \sin 2 \theta+B \cos 2 \theta)\right]$

Considering the relations (4) and (5), the equations (22) and (23) can be written, respectively:

$$
\begin{aligned}
& X_{f}=\lambda_{m}\left(2 C_{f}^{\prime} \sqrt{1+\kappa^{2}}\right)^{1 / 3}\left[\cos \theta+\frac{1}{2 \sqrt{1+\kappa^{2}}}(\cos 2 \theta-\kappa \sin 2 \theta)\right] \\
& Y_{f}=\lambda_{m}\left(2 C_{f}^{\prime} \sqrt{1+\kappa^{2}}\right)^{1 / 3}\left[\sin \theta+\frac{1}{2 \sqrt{1+\kappa^{2}}}(\sin 2 \theta+\kappa \cos 2 \theta)\right]
\end{aligned}
$$

and:

$$
\begin{aligned}
& X_{r}=\lambda_{m}\left(2 C_{r}^{\prime} \sqrt{1+\kappa^{2}}\right)^{1 / 3}\left[\cos \theta-\frac{1}{2 \sqrt{1+\kappa^{2}}}(\cos 2 \theta-\kappa \sin 2 \theta)\right] \\
& Y_{r}=\lambda_{m}\left(2 C_{r}^{\prime} \sqrt{1+\kappa^{2}}\right)^{1 / 3}\left[\sin \theta-\frac{1}{2 \sqrt{1+\kappa^{2}}}(\sin 2 \theta+\kappa \cos 2 \theta)\right]
\end{aligned}
$$

where:

$$
\kappa=\frac{k_{2}}{k_{1}}=\frac{B}{A}=\frac{\varphi+\frac{1}{2} \sin 2 \varphi}{\varphi-\frac{1}{2} \sin 2 \varphi} \tan \omega
$$

and:

$$
C_{r, f}^{\prime}=-\frac{\varepsilon z_{0} d c_{r, f} k_{1}}{\lambda_{m}}
$$

Fig. (2) illustrates the ratio $\kappa=k_{2} / k_{1}$ of the stress concentration factors versus the wedge angles $\varphi$ for angles of loading $\omega=1^{\circ}, 10^{\circ}, 45^{\circ}, 60^{\circ}, 80^{\circ}$.

For $\omega=0(\mathrm{~B}=0)$, concentrated vertical load at $O$ [13], the initial curve of the caustics and the respective parametric equations of the caustics, are:

$$
\begin{aligned}
& r_{0}=r=\left(2 C_{r, f} A\right)^{1 / 3} \\
& X_{f}=\lambda_{m}\left(2 C_{f} A\right)^{1 / 3}\left[\cos \theta+\frac{1}{2} \cos 2 \theta\right] \\
& Y_{f}=\lambda_{m}\left(2 C_{f} A\right)^{1 / 3}\left[\sin \theta+\frac{1}{2} \sin 2 \theta\right] \\
& X_{r}=\lambda_{m}\left(2 C_{r} A\right)^{1 / 3}\left[\cos \theta-\frac{1}{2} \cos 2 \theta\right] \\
& Y_{r}=\lambda_{m}\left(2 C_{r} A\right)^{1 / 3}\left[\sin \theta-\frac{1}{2} \sin 2 \theta\right]
\end{aligned}
$$

for $-\phi \leq \theta \leq+\phi$

For $\omega=\pi / 2(\mathrm{~A}=0)$, concentrated horizontal load at $O$ the initial curve of the caustics and the respective parametric equations of the caustics, are:

$$
\begin{aligned}
& r_{0}=r=\left(2 C_{r, f} B\right)^{1 / 3} \\
& X_{f}=\lambda_{m}\left(2 C_{f} B\right)^{1 / 3}\left[\cos \theta-\frac{1}{2} \sin 2 \theta\right]
\end{aligned}
$$




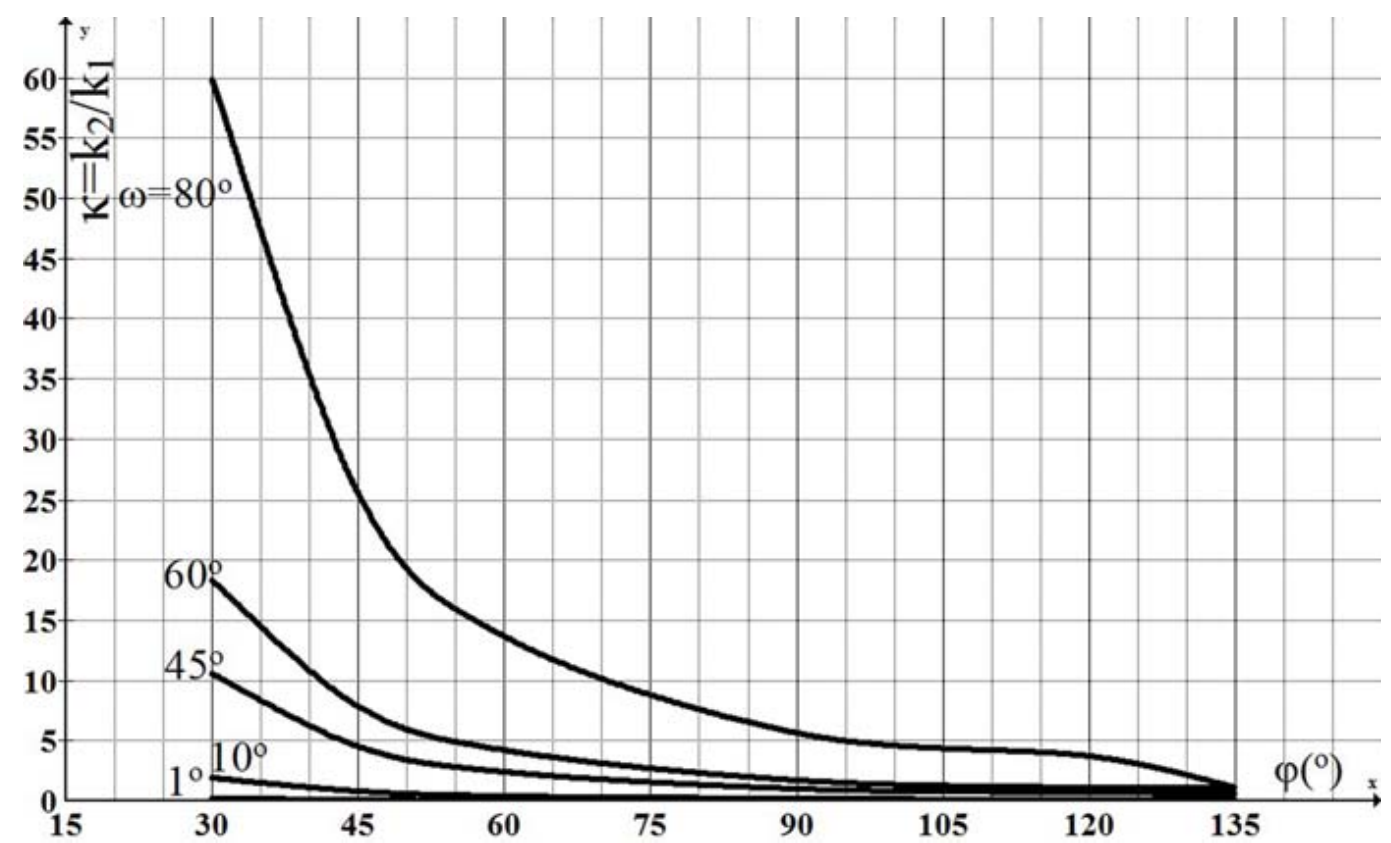

Fig. (2). Variation of the stress concentration factors ratio $k_{2} / k_{1} v s$ the wedge angles $\varphi$ for angles of loading $\omega=1^{\circ}, 10^{\circ}, 45^{\circ}, 60^{\circ}, 80^{\circ}$.

$Y_{f}=\lambda_{m}\left(2 C_{f} B\right)^{1 / 3}\left[\sin \theta+\frac{1}{2} \cos 2 \theta\right]$

$X_{r}=\lambda_{m}\left(2 C_{r} B\right)^{1 / 3}\left[\cos \theta+\frac{1}{2} \sin 2 \theta\right]$

$Y_{r}=\lambda_{m}\left(2 C_{r} B\right)^{1 / 3}\left[\sin \theta-\frac{1}{2} \cos 2 \theta\right]$

for $-\phi \leq \theta \leq+\phi$

For values of $\theta$ between $-\varphi$ and $+\varphi$, the caustic (r), from the rear face of the plate and the caustic (f), from the front face of the plate, are obtained around the point of the concentrated load $P$. Fig. (3) illustrates the shapes of the respective caustics for angle loading $\omega=45^{\circ}$ and for (a)wedge of $\varphi=45^{\circ}$, (b)wedge of $\varphi=90^{\circ}$, (c) wedge of $\varphi=120^{\circ}$ and (d) wedge of $\varphi=135^{\circ}$. The respective positions and the magnitude of the two caustics are depended on the values of the respective radii of the initial curves and the stress optical constants $c_{r}, c_{f}$. Also, Fig. (4) illustrates the shapes of the respective caustics around the point of the concentrated load for wedge of $\varphi=90^{\circ}$ (semi-infinite plate) and for angles of loading (a) $\omega=0^{\circ}$ (vertical load [13]), (b) $\omega=60^{\circ}$, (c) $\omega=80^{\circ}$ and (d) $\omega=90^{\circ}$ (horizontal load).

\section{EXPERIMENTAL SET-UP}

In order to verify the theoretical results of the concentrated load and the concentration factors evaluation at the apex of the wedges by the method of caustics, specimens made of Plexiglas (PMMA) were used. The thickness of the specimens was $d=3 \times 10^{-3} \mathrm{~m}$. The experimental set-up was simple, Fig. (5) [1-3]. A coherent divergent light beam from a He-Ne gas laser impinged on the face of the specimen close to apex of the wedge. Screen in front the specimen was placed at distance $z_{0}$ and parallel to specimen mid-plane. On this screen the caustics from reflected rays were formed and recorded by a camera. The optical set-up had $z_{0}=1.79 \mathrm{~m}$, $z_{i}=0.30 \mathrm{~m}$ and the magnification ratio $\lambda_{m}$ of the optical system was $\lambda_{m}=6.97$ (Rel. (14)). For a positive value of $z_{i}$ (the focus lying in front of the specimen) a double caustic is formed on the screen due to reflections from the front and the rear face of the specimen (caustics of Figs. 3, 5). The mechanical properties of the specimen material (Plexiglas) were: Poisson's ratio $v=0.38$ and elastic modulus $E=2.2 \mathrm{MPa}$ The stress-optical constant of the specimens material was $c_{f}=\frac{v}{E}=1.7 \times 10^{-10} \mathrm{~m}^{2} / \mathrm{N}$. The specimens were loaded by a uniform concentrated load at the apex of the wedges.

Fig. (6) illustrates the experimental reflected caustics (f) and ( $r$ ) which were carried out by divergent light beam, for a Plexiglas specimens of thickness $d=4 \times 10^{-3} \mathrm{~m}$. The loading angle was $\omega=0^{\circ}$ and the wedge angles were (a) $\varphi=45^{\circ}$, (b) $\varphi=90^{\circ}$ and (c) $\varphi=120^{\circ}$. Also, Fig. (7) illustrates the theoretical (a) and the experimental (b) reflected caustics (f) and (r) for a Plexiglas specimens of thickness $d=3 \times 10^{-3} \mathrm{~m}$. The loading angle was $\omega=25^{\circ}$ and the wedge angles was (a) $\varphi=135^{\circ}$, We can see good quality agreement between theoretical and experimental caustics. The relative position of the two caustics, (r) and (f), is depended on the values of the stress optical constants $c_{r}$ and $c_{f}$.

From Figs. $(3,4)$ it is appeared that for oblique loading the diameters of the caustic can not be calculated. In these cases the load $P$ and the concentration factors can be calculated from the opening displacement $L$ of the caustic (Fig. 3b). The horizontal and the vertical components of the opening displacement $L$ are given: 


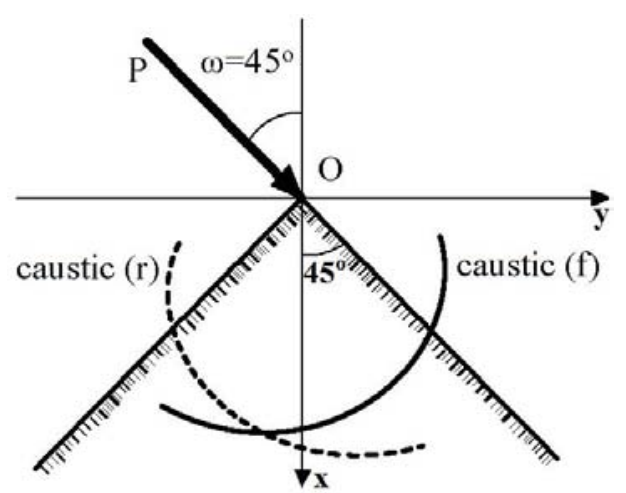

(a)

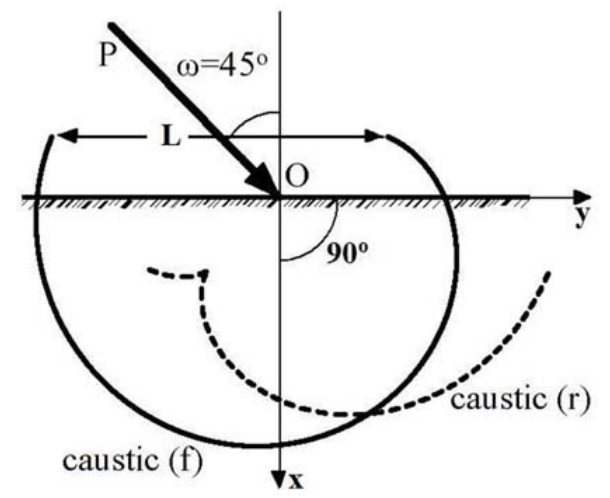

(b)

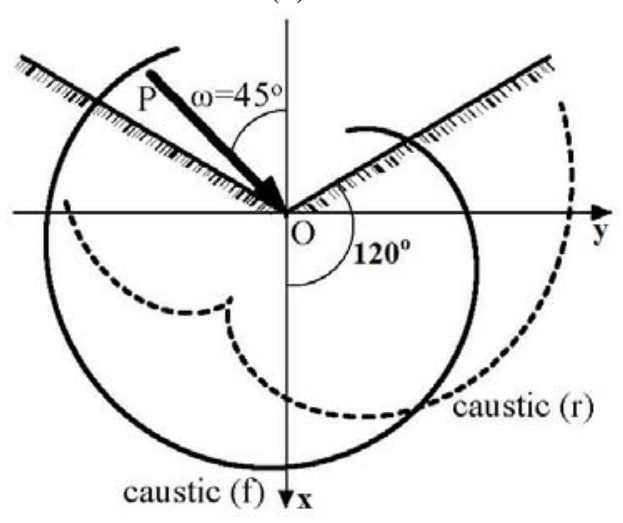

(c)

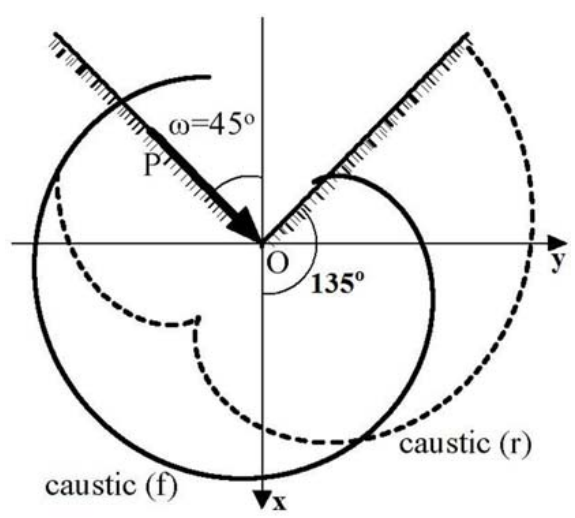

(d)

Fig. (3). Caustics (r) and (f) at the apex of wedge for angle of loading $\omega=45^{\circ}$ and for wedges of (a) $\varphi=45^{\circ}$, (b) $\varphi=90^{\circ}$, (c) $\varphi=120^{\circ}$ and (d) $\varphi=135^{\circ}$.

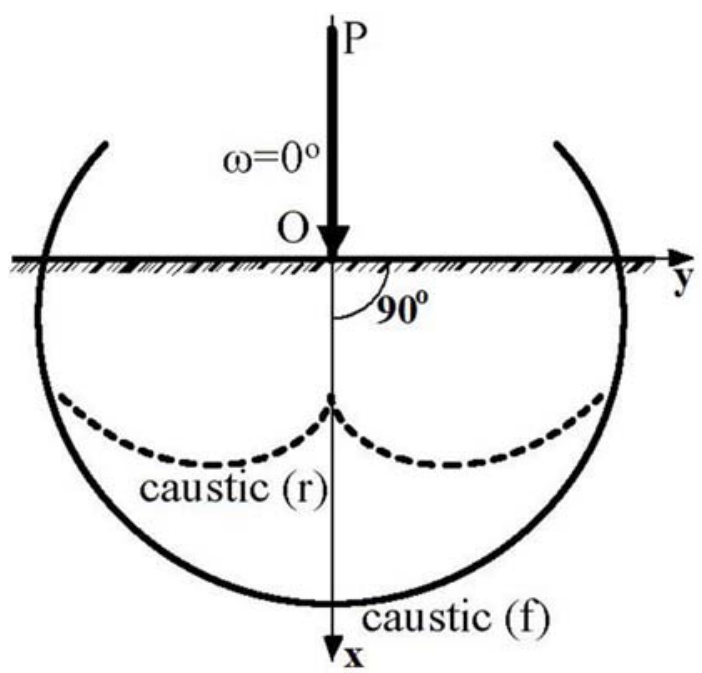

(a)

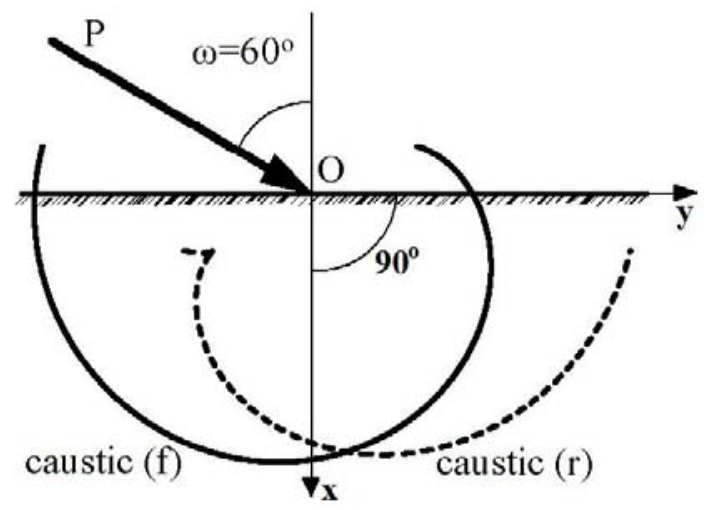

(b)

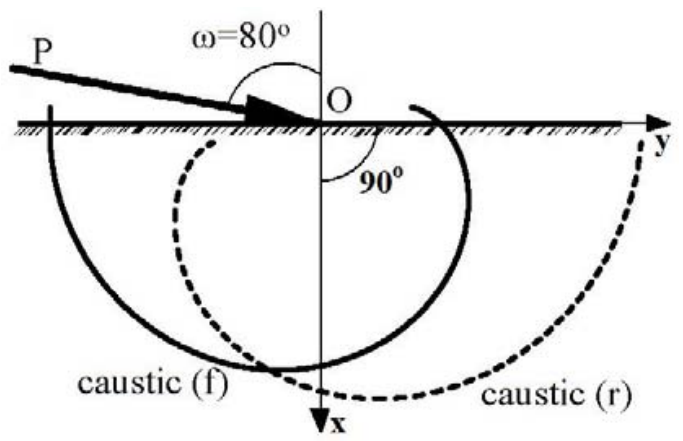

(c)

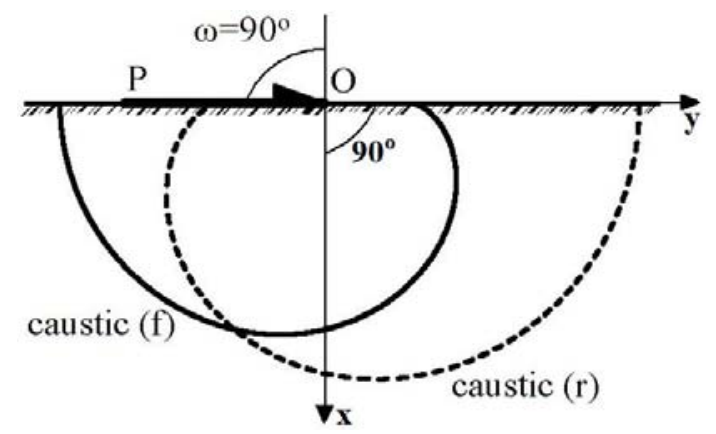

(d)

Fig. (4). Caustics (r) and (f) at the apex of wedge of angle $\varphi=90^{\circ}$ (semi-infinite plate) and for loading angles of (a) $\omega=10^{\circ}$ (about vertical force), (b) $\omega=60^{\circ}$, (c) $\omega=80^{\circ}$ and (d) $\omega=90^{\circ}$ (horizontal force). 


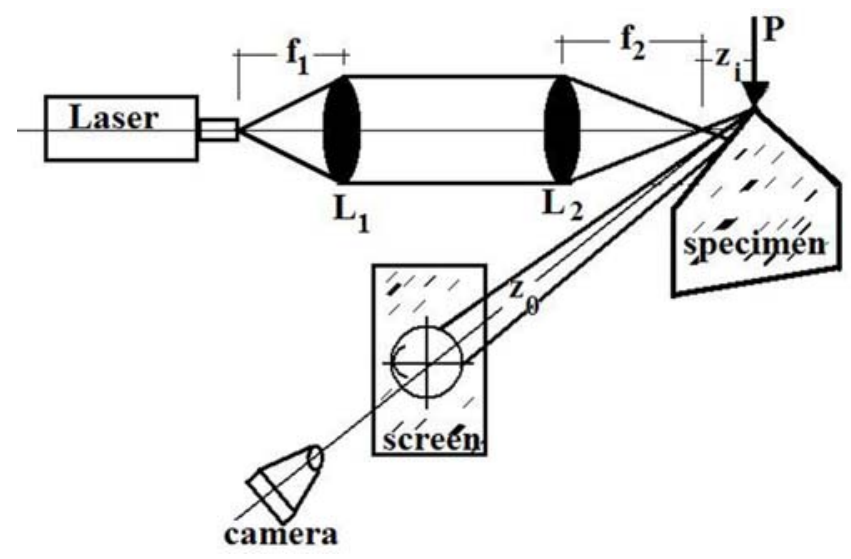

Fig. (5). The optical set-up for reflected caustics. $L_{1}, L_{2}$ lenses of focus-distances $f_{1}, f_{2}$, respectively.

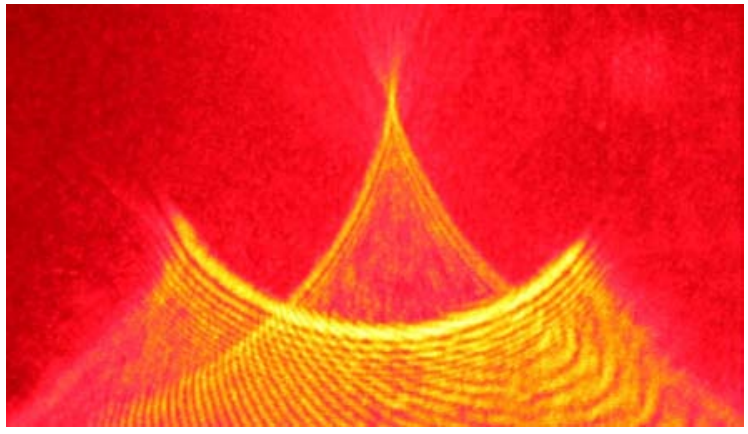

(a)

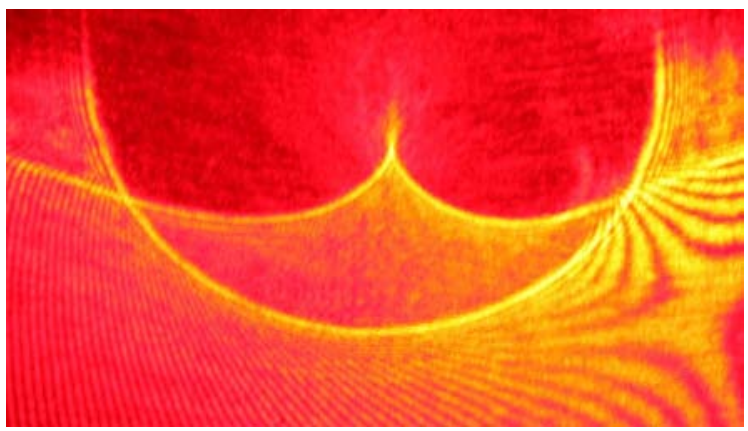

(b)

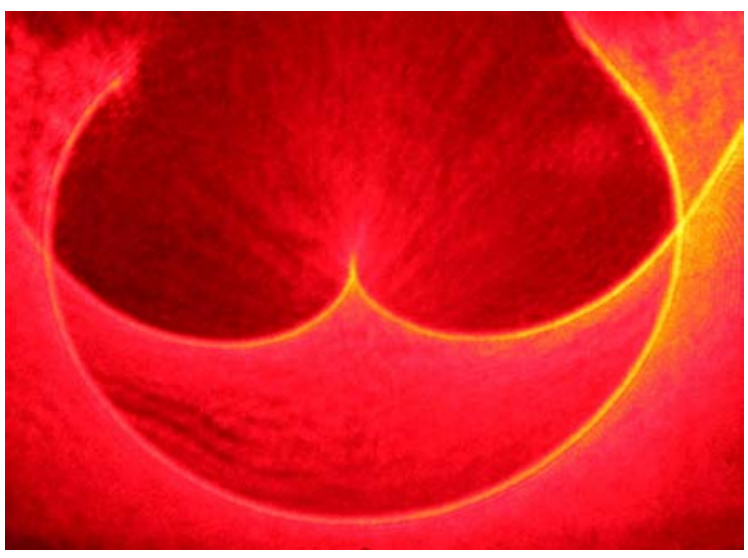

(c)

Fig. (6). Experimental caustics (r) and (f) at the apex of wedge for angle of loading $\omega=0^{\circ}$ and for wedges of (a) $\varphi=45^{\circ}$, (b) $\varphi=90^{\circ}$ and (c) $\varphi=120^{\circ}$.

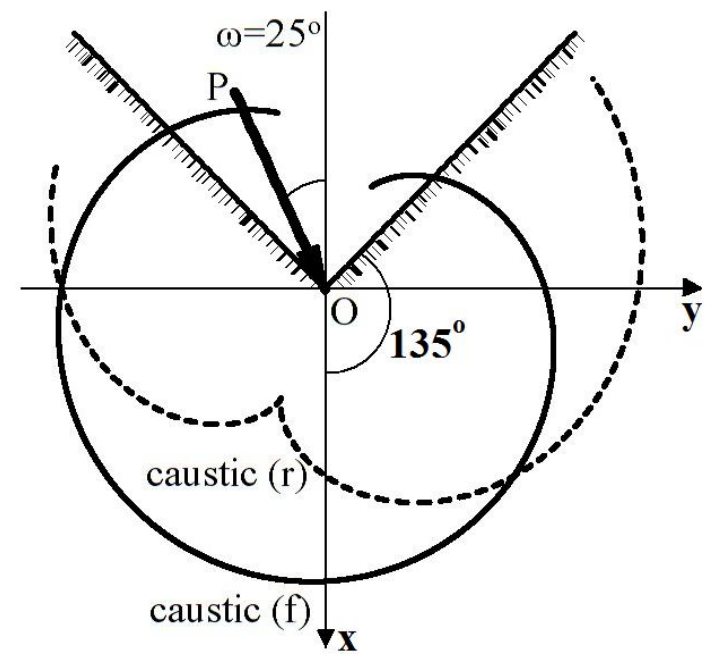

(a)

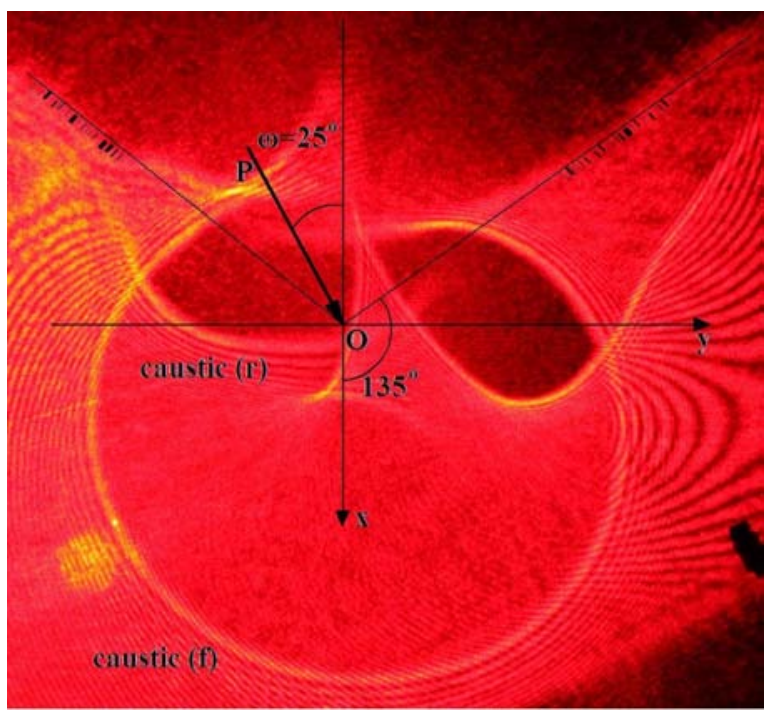

(b)

Fig. (7). Theoretical (a) and experimental (b) caustics (r) and (f) at the apex of wedge for angle of loading $\omega=25^{\circ}$ and for wedge of $\varphi=135^{\circ}$.

$$
\begin{aligned}
L_{H} & =\left.Y_{f}\right|_{\theta=\varphi}-\left.Y_{f}\right|_{\theta=-\varphi} \\
& =2 \lambda_{m}\left(2 C_{f} \sqrt{A^{2}+B^{2}}\right)^{1 / 3}\left[\sin \varphi+\frac{A}{2 \sqrt{A^{2}+B^{2}}} \sin 2 \varphi\right]
\end{aligned}
$$

The vertical component of the opening displacement $\mathrm{L}$ is given:

$$
\begin{aligned}
L_{V} & =\left.X_{f}\right|_{\theta=-\varphi}-\left.X_{f}\right|_{\theta=\varphi} \\
& =2 \lambda_{m}\left(2 C_{f} \sqrt{A^{2}+B^{2}}\right)^{1 / 3}\left[\frac{B}{2 \sqrt{A^{2}+B^{2}}} \sin 2 \varphi\right]
\end{aligned}
$$

and the opening displacement $\mathrm{L}$ of the caustic is:

$$
L=\sqrt{L_{H}^{2}+L_{V}^{2}}=2 \lambda_{m}\left(2 C_{f} \sqrt{A^{2}+B^{2}}\right)^{1 / 3} R
$$

where: 
$R=\left[\left(\sin \varphi+\frac{A}{2 \sqrt{A^{2}+B^{2}}} \sin 2 \varphi\right)^{2}+\left(\frac{B}{2 \sqrt{A^{2}+B^{2}}} \sin 2 \varphi\right)^{2}\right]^{1 / 2}$

By comparing equations (17) and (36) the oblique concentrated load $P$ is calculated by the relation:

$P=\frac{L^{3}}{16 z_{0} d \lambda_{m}^{2} c_{f}} \frac{1}{R^{3} \sqrt{A^{2}+B^{2}}}$

Because of the $P$ is the load per unit thickness, the total load is:

$Q=P d=\frac{L^{3}}{16 z_{0} \lambda_{m}^{2} c_{f}} \frac{1}{R^{3} \sqrt{A^{2}+B^{2}}}$

The experimental stress concentration factors $k_{1}^{e x}, k_{2}^{e x}$, according to relations (4), (5) and (39), can be calculated:

$$
\begin{aligned}
& k_{1}^{e x}=\frac{L^{3} \cos \omega}{16 z_{0} \lambda_{m}^{2} c_{f}\left(\varphi+\frac{1}{2} \sin 2 \varphi\right) R^{3} \sqrt{A^{2}+B^{2}}} \\
& k_{2}^{e x}=\frac{L^{3} \sin \omega}{16 z_{0} \lambda_{m}^{2} c_{f}\left(\varphi-\frac{1}{2} \sin 2 \varphi\right) R^{3} \sqrt{A^{2}+B^{2}}}
\end{aligned}
$$

Considering the relations (4), (5) and (40), (41), a correction factors $f_{1,2}$ can be calculated [21]:

$$
f_{1,2}=\frac{k_{1,2}^{e x}}{k_{1,2}}
$$

\section{ESTIMATION OF THE STRESSES $\Sigma$ IJ}

On the horizontal plane nn at a distance $x_{0}$ from the apex of the wedge, the Cartesian stress components at any point M (Fig. 1) are calculated from the polar stress component $\sigma_{r r}:$

$$
\begin{aligned}
\sigma_{x x} & =\sigma_{r r} \cos ^{2} \theta=-P A \frac{\cos ^{3} \theta}{r}+P B \frac{\sin \theta \cos ^{2} \theta}{r} \\
& =-k_{1} \frac{\cos ^{4} \theta}{x_{0}}+k_{2} \frac{\sin \theta \cos ^{3} \theta}{x_{0}} \\
\sigma_{y y} & =\sigma_{r r} \sin ^{2} \theta=-P A \frac{\cos \theta \sin ^{2} \theta}{r}+P B \frac{\sin ^{3} \theta}{r} \\
& =-k_{1} \frac{\cos ^{2} \theta \sin ^{2} \theta}{x_{0}}+k_{2} \frac{\sin ^{3} \theta \cos \theta}{x_{0}} \\
\tau_{x y} & =\sigma_{r r} \sin \theta \cos \theta=-P A \frac{\sin \theta \cos ^{2} \theta}{r}+P B \frac{\sin ^{2} \theta \cos \theta}{r} \\
& =-k_{1} \frac{\sin \theta \cos 3}{x_{0}}+k_{2} \frac{\sin ^{2} \theta \cos ^{2} \theta}{x_{0}}
\end{aligned}
$$

with:

$r=x_{0} / \cos \theta=r_{0} / \cos \theta$

For a horizontal plane nn close to the apex of the wedge at a distance equals to the radius of the initial curve of the caustic, $x_{0}=r_{0}$, and by substituting relations (21), (36) and (38) into equations (43)-(45), the components of stress are obtained from the opening displacement of the caustic:

$$
\begin{gathered}
\sigma_{x x}=-\frac{L^{2}}{8 z_{0} d \lambda_{m} c_{f} R^{2} \sqrt{A^{2}+B^{2}}}\left(A \cos ^{4} \theta-B \sin \theta \cos ^{3} \theta\right) \\
\sigma_{y y}=-\frac{L^{2}}{8 z_{0} d \lambda_{m} c_{f} R^{2} \sqrt{A^{2}+B^{2}}}\left(A \cos ^{2} \theta \sin ^{2} \theta-B \sin ^{3} \theta \cos \theta\right) \\
\tau_{x y}=-\frac{L^{2}}{8 z_{0} d \lambda_{m} c_{f} R^{2} \sqrt{A^{2}+B^{2}}}\left(A \sin \theta \cos ^{3} \theta-B \sin ^{2} \theta \cos ^{2} \theta\right)
\end{gathered}
$$

for values of $\theta$ between $-\varphi$ and $+\varphi$.

Fig. (8) illustrates the distribution of the non-dimensional components of stress, (Eqs (47)-(49)):

$\sigma_{i j} \frac{M}{L^{2}}=f(\theta) \quad$ with $\quad M=-8 z_{0} d \lambda_{m} c_{f} R^{2} \sqrt{A^{2}+B^{2}}$

on the horizontal plane at distance $r_{0}$ (singular region of concentrated load) from the wedge apex, (Fig. 1), for wedges of angle up to $\varphi=3 \pi / 4$ and of angle of loading a) $\omega=0^{\circ}$, b) $\omega=45^{\circ}$ and c) $\omega=90^{\circ}$.

\section{EXPERIMENTAL RESULTS AND DISCUSSION}

In order to verify the theoretical results of the concentrated load and the concentration factors evaluation at the apex of the wedges by the experimental method of caustics, specimens made of Plexiglas (PMMA) and the experimental set-up of Fig. (5) were used. Figs. $(\mathbf{6}, 7)$ illustrate the reflected caustics (f) and (r) which were formed at the apex of the wedges. From the opening displacement $(L)$ of the caustics (f) (Fig. 3b) the concentred load $P$ (or $Q=P d$ ) and the concentrations factors $k_{1}^{e x}$ and $k_{2}^{e x}$ were calculated by the Eqs (38)-(41).

Fig. (9) illustrates the experimentally calculated load $Q$ (by Eq. (39)) from the opening displacements $L$ of the caustics (f), (Fig. 6), for wedges angles $\varphi=45^{\circ}, 90^{\circ}$ and $120^{\circ}$ and loading angle $\omega=0^{\circ}$ (vertical concentrated load). As it can be observed that the calculated load $Q$ depends on the opening displacement $(L)$ of the caustic. The opening displacement $(L)$ of the caustic depends on the wedge angles and the size of the initial curve $\left(r_{0}\right)$. The size of the initial curve is smaller than the theoretical one because of the boundary deformation of the specimen. The variation of the load with the theoretical opening displacement is parabolic 


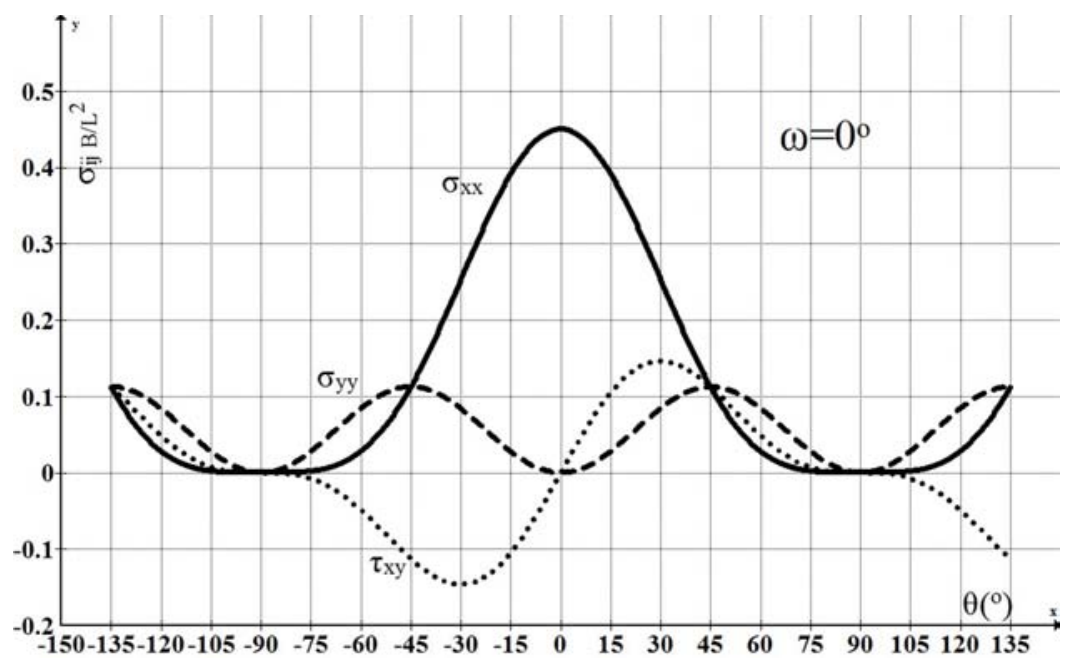

(a)

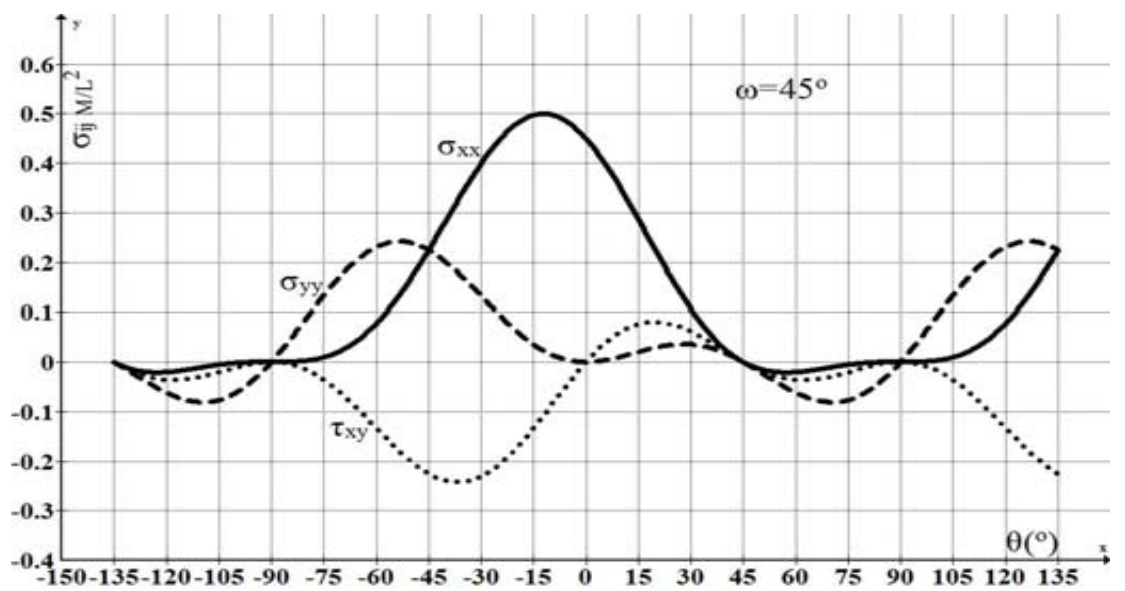

(b)

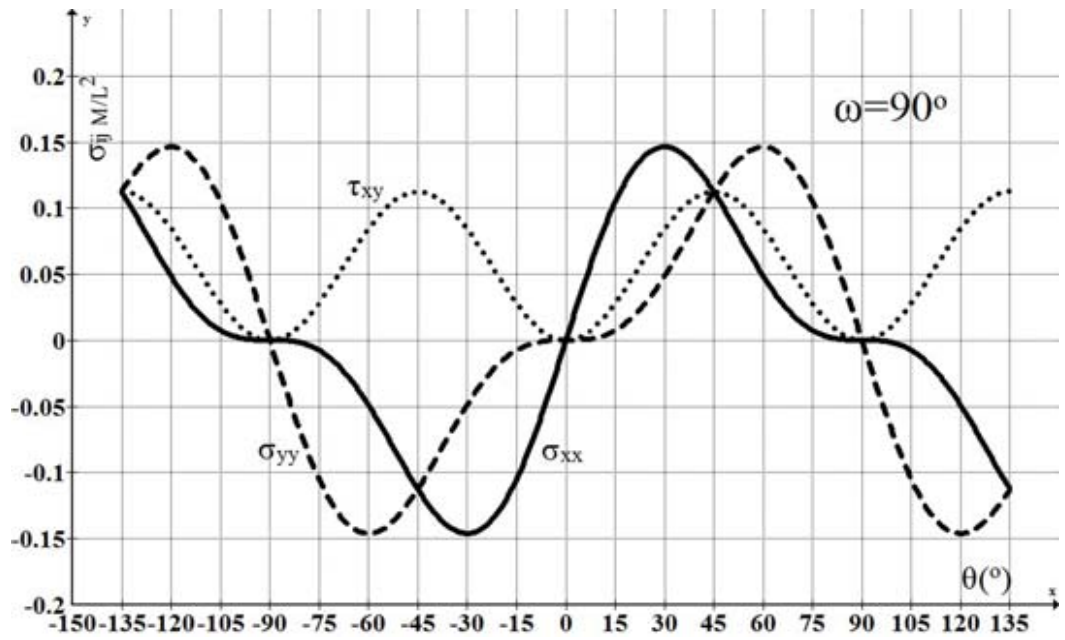

(c)

Fig. (8). The stress components variation versus $\theta$ for wedge angles up to $\varphi=3 \pi / 4$ and for loading angle a) $\left.\omega=0^{\circ}, \mathbf{b}\right) \omega=45^{\circ}$ and c) $\omega=90^{\circ}$.

(Rel. (39)). We can see a good agreement between the theoretical variation and experimentally calculated values based on the caustics (Fig. 9).
Fig. (10) illustrates the variation of the experimental concentration factor $k_{1}^{e x}$ which was calculated from the 
experimental caustics of Fig. (6). As it can be observed that the concentration factor increases as the wedge angles decrease. The variation of the theoretical stress concentration $k_{1}$ (Rel. (4)) is linear with the load. We can see a good agreement between the theoretical variation and experimentally calculated values based on the caustics (Fig. 10).

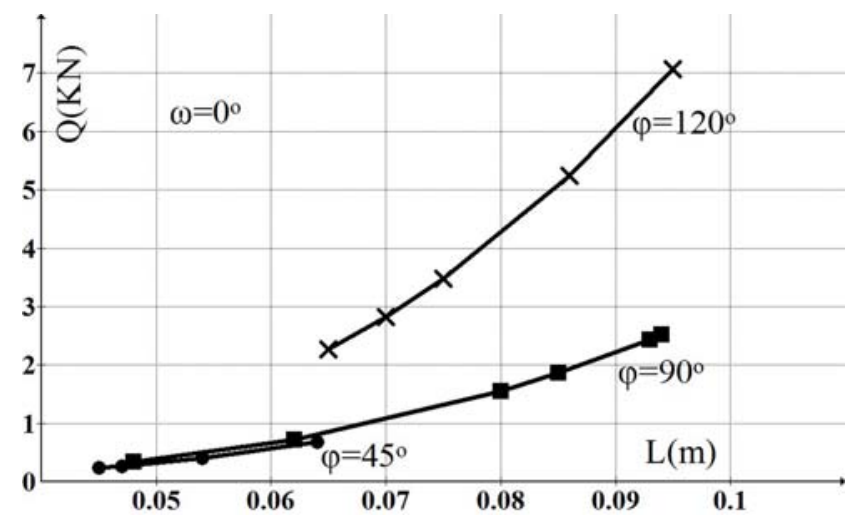

Fig. (9). Variation of the experimentally calculated load $Q v s$ the caustics opening displacement $\mathrm{L}$ for wedge angles $\varphi=45^{\circ}, 90^{\circ}$ and $120^{\circ}$ and loading angle $\omega=0^{\circ}$.

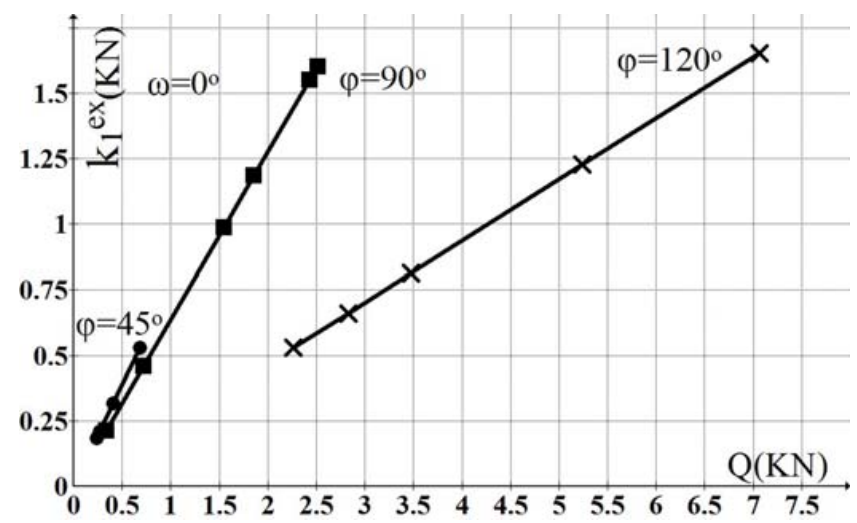

Fig. (10). Variation of the concentration factor $k_{1}^{e x} v s$ the load $Q$ for wedge angles $\varphi=45^{\circ}, 90^{\circ}$ and $120^{\circ}$ and loading angle $\omega=0^{\circ}$.

Fig. (11) illustrates the variation of the experimental concentration factors $k_{1}^{e x}, k_{2}^{e x}$ and the ratio of concentration factors $\kappa=k_{2}^{e x} / k_{1}^{e x}$ which were calculated from the experimental caustics of Fig. (7b) (wedge angle $\varphi=135^{\circ}$ and loading angle $\omega=25^{\circ}$ ). As it can be observed that the concentration factors increase as the load increases, while the concentration factors ratio is remain constant. The concentration factors $k_{1}^{e x}, k_{2}^{e x}$ are depend on the load $P$ (Rels (4) and (5)), while the ratio $\kappa=k_{2}^{e x} / k_{1}^{e x}$ is independent on the load (Rel. (26)). We can see good agreement between the theoretical variation and the experimental values based on the caustics (Fig. 11).

\section{CONCLUSIONS}

An experimental method of caustics has been proposed to calculate experimentally the load and the concentration factor at the apex of the wedges. A detail analysis of the reflected caustics is proved for the study the contact problems. Using this experimental analysis of the caustics, stress information may be obtained in singular points of the contact problems. It can be concluded that:

The concentrated load, the concentration factors and the stress variation at the singular apex of the wedges may be experimentally calculated by the experimental method of the caustics. The experimental method of caustics which is a powerful tool for discussing fields around a crack, is also a powerful method for the contact problems and wedges with concentration loads. Using this experimental method the existing wedge theory may be reviewed with measurements and taking into consideration other influential parameters such as the wedge angles and the loading angles.

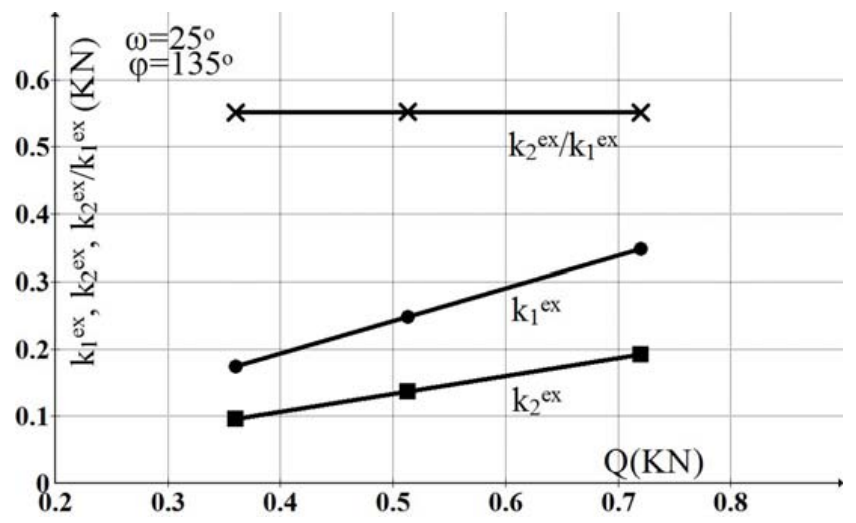

Fig. (11). Variation of concentration factors $k_{1}^{e x}, k_{2}^{e x}$ and the ratio $\kappa=k_{2}^{e x} / k_{1}^{e x} v s$ the load $Q$ for wedge angle $\varphi=135^{\circ}$ and loading angle $\omega=25^{\circ}$.

\section{ACKNOWLEDGEMENT}

Declared none.

\section{CONFLICT OF INTEREST}

The authors confirm that this article content has no conflicts of interest.

\section{REFERENCES}

[1] P. S. Theocaris, "Local yielding around a crack-tip in Plexiglas". Journal of Applied Mechanics, Trans. ASME, vol. 37, pp. 409-415, 1970.

[2] J. F. Kalthoff, "Shadow optical method of caustics", in Handbook on Experimental Mechanics, A. S. Kobayashi, Ed. USA: PrenticeHall, 1987, chapt. 9, pp. 430-500.

[3] G. A. Papadopoulos, Fracture Mechanics, The Experimental Method of Caustics and the Det.-Criterion of Fracture, G.A. Papadopoulos Ed., London: Springer-Verlag 1993.

[4] S. P. Timoshenko and J. N. Goodier, Theory of Elasticity, New York: McGraw Hill, 1970.

[5] A. Flamant, "De l'influence sur la flexion des poutres de la position superficielle de la charge" Annales des Ponts et Chaussees, vol. VI, pp. 228-242, 1893.

[6] J. Boussinesq, "Perturbations locales que produit audessous d'ell une forte charge reparie uniformement l'ong d'une droit normale aux debords a la surface superieure d'une poutre rectangulaire et de longueur indefinie posee de champ soit sur un sol horizontal, soit sur deux appuis traversaux equidistants de la charge"', Comptes Rendus de l'Académie des Sciences, vol. 114, pp. 1510- 1520, 1892.

[7] P. S. Theocaris, "Stress-singularities due to uniformly distribution loads along straight boundaries", International Journal of Solids and Structures, vol. 9, pp. 655-670, 1973.

[8] P. S. Theocaris, "Stress-singularities at concentrated loads", Experimental Mechanics, vol. 13, pp. 511-518, 1973. 
[9] G. A. Papadopoulos, "Experimental estimation of the load distribution in bearings by the method of caustics", Experimental Mechanics, vol. 44, pp. 440-443, 2004.

[10] G. A. Papadopoulos, "Experimental study of the load distribution in bearing by the method of caustics and the photoelasticity method", The Journal of Strain Analysis for Engineering Design, vol. 40, pp. 357-365, 2005.

[11] V. A. Spitas, G. K. Sfantos, Th. Costopoulos, C. A. Spitas, and G. A. Papadopoulos, "Numerical and experimental Analysis of load sharing in multiple gear tooth contact". $7^{\text {th }}$ National Congress on Mechanics, June 24-26, 2004, Chania, Greece, vol. I, pp. 232-237.

[12] V. Spitas, G. A. Papadopoulos, C. Spitas and T. Costopoulos, "Experimental investigation of load sharing in multiple gear tooth contact using the stress-optical method of caustics". Strain, vol. 47, pp. e227-e233, 2009.

[13] G. A. Papadopoulos, Study of concentrated vertical load at the apex of a wedge by the experimental method of caustics and photoelasticity", Advances and Applications in Mechanical Engineering and Technology (AAMET), vol. 3, pp. 59-81, 2011.

[14] V. M. Aleksadrov and D. A. Pozharskii, "Problems of a cut in a three-dimensional elastic wedge", Journal of Applied Mathematics and Mechanics, vol. 70, pp. 483-492, 2006.
[15] A. R. Shahani, "Some problems in the antiplane shear deformation of bimaterial wedges", International Journal of Solids and Structures, vol. 42, pp. 3093-3113, 2005.

[16] Y. C. Gao, "Large strain analysis of a rubber wedge compressed by a line load at its tip", International Journal of Engineering Science, vol. 36, pp. 831-842, 1998.

[17] B. G. Badalouka and G. A. Papadopoulos, "Range of applicability of the method of caustics around holes". Strain, vol. 42, pp. 283290, 2006.

[18] D. N. Pazis, Z. Agioutantis and S. K. Kourkoulis, S. K., 2010, "The optical method of reflected caustics applied for a plate with a central hole: Critical points and limitations". Strain, vol 47, pp. 489-498, 2010.

[19] A. Bacic, D. Semenski and S. Jecic, "The caustics method in the contact problems of anisotropic materialas", EPJ Web of Conference, vol. 6, pp.1-8, 2010.

[20] M. Born and E. Wolf, Principles of Optics, London: Pergamon, 1970 .

[21] P. S. Theocaris and G. A. Papadopoulos, "The influence of geometry of edge-cracked plates on $\mathrm{K}_{\mathrm{I}}-$ and $\mathrm{K}_{\mathrm{II}}-$ components of the stress intensity factor", Journal of Physics D: Applied Physics, vol. 17, pp. 2339-2349, 1984.

(C) G.A. Papadopoulos; Licensee Bentham Open.

This is an open access article licensed under the terms of the Creative Commons Attribution Non-Commercial License (http://creativecommons.org/licenses/by-nc/3.0/) which permits unrestricted, non-commercial use, distribution and reproduction in any medium, provided the work is properly cited. 